DOI:10.2478/rrlm-2019-0031

\title{
Cynomorium Songaricum may protect against spermatogenic damage caused by cyclophosphamide in SD rats
}

\author{
Xiaoxiao Han ${ }^{1 \#}$, Rongsheng Zhou ${ }^{2 \#}$, Wei Zheng ${ }^{3 \#}$, Xitao Wang ${ }^{2,4}$, Shuyan $\mathrm{Mao}^{5}$, \\ Zhenbei $\mathrm{Li}^{6}$, Lin $\mathrm{Hao}^{2}$, Zhenduo Shi², Bo Chen ${ }^{2}$, Zhiguo Zhang ${ }^{2}$, Yijuan $\mathrm{Cao}^{6}$, \\ Dianjun $\mathrm{Yu}^{7}$, Kun Pang ${ }^{2,8^{*}}$, Conghui Han ${ }^{2,8^{*}}$
}

1. Department of Reproductive Medicine, Shanghai First Maternity and Infant Hospital. No. 2699

Gaoke West Road, Pudong District, Shanghai, China.

2. Department of Urology, Xuzhou Central Hospital, Jiangsu Xuzhou Jiefang South Road, No. 199, Jiangsu, China.

3. Department of Urology, Kiangwu Hospital. Macao, China.

4. Department of Reproductive Center, Xuzhou Central Hospital, Jiangsu Xuzhou Jiefang South Road, No. 199, Jiangsu, China.

5. Medical school, Southeast University. No. 2, Sipai Building, Xuanwu District, Nanjing, China.

6. Department of Pathology, Xuzhou Central Hospital, Jiangsu Xuzhou Jiefang South Road, No. 199, Jiangsu, China.

7. Department of Urology, Li Huili Hospital, No. 57, Xingning Road, Jiangdong District, Ningbo, Zhejiang, China

8. The third affiliated hospital of Soochow University, No.185, Juqian Street, Changzhou City, Jiangsu Province China

\begin{abstract}
Aim: The aim of the study is to investigate the effect of Cynomorium songaricum (CS) on the damage caused by cyclophosphamide (CP) in SD rats. Methods: Rats with CP-induced oligoasthenospermia were treated with different concentration of CS. Testicle weight, epididymal sperm count (ESC), sperm motility, and serum testosterone were analyzed, and expression levels of Thy1, Oct4, PLZF, C-kit, and GDNF were detected in testis tissues. Transmission electron microscopy (TEM) was performed to observe the effect of CS on the spermatogenic damage by CP. Results: Compared with the CP group, there were significant differences in testicle weight, ESC, and sperm motility

*Corresponding author: Kun Pang and Conghui Han, Department of Urology, Xuzhou Central Hospital, Jiangsu Xuzhou Jiefang South Road, No. 199, Jiangsu, China. E-mail: pakn1987@163.com

"Xiaoxiao Han, Rongsheng Zhou and Wei Zheng contributed equally to this work
\end{abstract}


$(p<0.05)$ observed in all concentrations of CS and CP+VitE groups $(p<0.05)$. There were no significant differences in serum testosterone among the 6 groups ( $p>0.05)$. The qPCR results revealed a significant difference in Thyl, Oct4, PLZF and GDNF expression between the CP group and CS group $(p<0.05)$, but there was no significant difference in C-kit between the two groups ( $p>0.05$ ). The damage of CP was cured by CS observed under TEM. Conclusion: CS can increase sperm counts in the epididymis and improve sperm motility and has a therapeutic effect on the spermatogenic damage caused by $C P$ in $S D$ rats.

Keywords: Cynomorium Songaricum, spermatogenic damage, cyclophosphamide, epididymal sperm count, sperm motility

Received: 19 th December 2018; Accepted: 11 th July 2019; Published: $14^{\text {th }}$ July 2019

\section{Introduction}

Oligospermia and asthenospermia in men are important factors leading to infertility (1). Thus, improving sperm quality is a critical issue in modern reproductive research.

There are many causes of decreased number of spermatozoa and reduced activity, including reproductive tract infections, semen liquefaction, and endocrine hormone disorders. There may be limited improvement with the use of anti-infective and anti-oxidant treatments. Chinese medicines have several unique effects on this disease. Cynomorium songaricum (CS) is a traditional kidney medicine that affects the liver, kidney, and large intestine. CS contains organic acids, flavonoids, steroids, triterpenes, and amino acids. CS has been reported to enhance immune function, scavenge free radicals, and function as an anti-oxidant that impacts aging, and CS has also been shown to reduce hypoxia, stress, and fatigue. CS contains a variety of essential trace elements such as $\mathrm{Cu}, \mathrm{Mn}$, and $\mathrm{Mi}$ as well as high Fe and $\mathrm{Zn}$ content (2). Yang et al. (3) showed that the expression of glial cell line-derived neurotrophic factor (GDNF) mRNA and protein as well as the number of epididymal spermatozoa and the absolute weight of testis are increased after CS administration. The authors also reported a reduction in the number of abnormal sperm and improved sperm motility following CS treat- ment (4). CS also increases the testicular weight of immature male Wistar rats (5). The aim of this study was to investigate the effects of CS on the number and activity of spermatozoa upon treatment of cyclophosphamide (CP)-related oligospermia in rats. Initial body weight (IBW), final body weight (FBW), testicle weight (TW), epididymal sperm count (ESC), sperm motility (SM), and serum testosterone (ST) were measured, and the expression levels of Thy1, Oct4, PLZF, C-kit, and GDNF were detected in testis tissue. Transmission electron microscopy (TEM) was performed to observe the effect of CS on the spermatogenic damage caused by cyclophosphamide $(\mathrm{CP})$.

\section{Materials and methods}

\section{Animals and Ethics}

This study used 36 SPF (Skatepark Formula ${ }^{\mathrm{TM}}$ ) grade Sprague Dawley® Rats (SD) healthy male 8 -week-old rats. The rats weighed approximately $215 \pm 5 \mathrm{~g}$ and the study was provided by the Experimental Animal Center of Xuzhou Medical University (SYXK (Su) 2017-0011).

\section{Drugs}

CP was obtained from Jiangsu Hengrui Pharmaceutical Co., Ltd. (Chinese medicine quasi-word H32020856, $0.1 \mathrm{~g} /$ support, powder). The CS was produced by Jiangsu Bozhou. Preparation 
of CS: Take $100 \mathrm{~g}$ dry CS and $1000 \mathrm{ml}$ saline, soak for $60 \mathrm{~min}$, boil for $30 \mathrm{~min}$, and filter with 2 layers of filter cloth. Next, add $500 \mathrm{ml}$ saline, soak for $30 \mathrm{~min}$, boil for $20 \mathrm{~min}$, and filter with 2 layers of filter cloth. Then, add $500 \mathrm{ml}$ saline, soak for $30 \mathrm{~min}$, boil for $10 \mathrm{~min}$, and filter with 2 layers of filter cloth. Combine all 3 filtrates, and filter with 4 layers of filter cloth to remove fried impurities. The filtrate is then added to $80 \sim 90^{\circ} \mathrm{C}$ water to a specific concentration (every $1 \mathrm{ml}$ of liquid CS equivalent to $0.5 \mathrm{~g}$ dry CS dose), and the reserve material is stored in the cold.

\section{Grouping and treatment methods}

The 36 SD rats were fed for 1 week of adaptation and then treated with drugs via intragastric administration. The rats were divided into 6 groups according to the random concentration table method, including a control group (blank control group) and $\mathrm{CP}, \mathrm{CP}+\mathrm{LCS}$ (low concentrations of $\mathrm{CS}), \mathrm{CP}+\mathrm{MCS}$ (medium concentrations of $\mathrm{CS}$ ), $\mathrm{CP}+\mathrm{HCS}$ (high concentrations of $\mathrm{CS}$ ) groups and a $\mathrm{CP}+$ VitE group. All SD rats except the control group were treated with CP $(30 \mathrm{mg} / \mathrm{kg}$ day) intraperitoneally for 5 days according to the literature (6). After 4 weeks of normal feeding, the rats in the $\mathrm{CP}+\mathrm{LCS}, \mathrm{CP}+\mathrm{MCS}$ and $\mathrm{CP}+\mathrm{HCS}$ groups were treated with CS at low $(0.5 \mathrm{~g} / \mathrm{kg} \cdot-$ day), medium (1 g/kg·day), and high (2 g/ $\mathrm{kg} \cdot-$ day) concentrations, and the $\mathrm{CP}+\mathrm{VitE}$ group was treated with Vitamin E (50 mg/kg.day) injection. There were no deaths during the intubation model.

\section{Detection of IBW, FBW, TW, ESC, SM, and ST in rats}

After 10 weeks, $10 \%$ chloral hydrate was used to anesthetize the rats. The testes and epididymis were collected, and the epididymis was placed at $37.5^{\circ} \mathrm{C}$ in salt solution in an Eppendorf (EP) tube. Smear observation was used to record sperm motility and sperm count in each group, and the testes of each group were weighed and recorded. A 200- $\mu 1$ sample of venous blood was collected from the abdominal aorta peripheral vein. Siemens ADVIA Centaur CP automatic chemiluminescence immunoassay analyzer and test kit for testosterone of Siemens Medical Diagnostics Co., Ltd were used to measure ST. A blood cell count plate was used to measure the ESC. The direct sperm count, non-linear advance sperm count, and immobile sperm count were measured to evaluate SM. The testicles were observed on 400X microscope with a hematoxylin-eosin (HE) staining.

\section{Real-time PCR}

The expression of Thy1, Oct4, PLZF, C-kit and GDNF in testis tissues were compared in the CP and $\mathrm{CP}+\mathrm{LCS}$ groups. Thy1, Oct4, PLZF, C-kit and GNDF primers were designed using Primer 5.0 software (Table 1). The house-keeping gene $\beta$-actin was used as an internal reference. Amplification conditions: $94^{\circ} \mathrm{C} 15 \mathrm{~min} ; 94^{\circ} \mathrm{C} 10 \mathrm{~s}$, $55^{\circ} \mathrm{C} 20 \mathrm{~s}, 72^{\circ} \mathrm{C} 20 \mathrm{~s}, 45$ cycles; $72^{\circ} \mathrm{C} 5 \mathrm{~min}$. The relative expression of each gene was calculated by fluorescence quantitative analysis using the $2^{-\Delta \Delta \mathrm{Ct}}$ method.

\section{Western blot analysis}

Western blot was performed to quantify the expression of spermatogonia-related proteins such as Thy1, Oct4, PLZF, C-kit and GDNF. Cells from the CP and CP+LCS groups were used. The BCA approach was used to measure total protein concentration. Proteins (30-50 mg) were resolved on a $10 \%$ Bis-Tris gradient SDS-PAGE gel under reducing conditions, transferred onto a polyvinylidene fluoride (PVDF) membrane and blocked with 5\% skim milk for $1 \mathrm{~h}$. Membranes were incubated with primary antibodies (Thy1, Oct4, PLZF, C-kit and GDNF) and glyceraldehyde-3-phosphate dehydrogenase (GAPDH) antibody at room temperature for $2 \mathrm{~h}$. Membranes were washed with TBST 4 times ( 8 minutes each) and incubated with fluorescein-linked sec- 
ondary antibodies at room temperature for 1.5 h. Next, membranes were washed with TBST another 4 times ( 8 minutes each), and signal detection was carried out using the Pierce ${ }^{\mathrm{TM}}$ ECL Western Blotting Substrate Kit (Thermo Fisher Sci. Co, Ltd, America) and X-ray film.

\section{TEM observation}

A small amount of little testicular tissue was taken from the CP group and CP+LCS group and fixed in $2.5 \mathrm{ml}$ of $1 \%$ glutaraldehyde precooled to $4^{\circ}$ after incubation with strong acid. The tissue was dehydrated and embedded with Epon812 resin. Semi-thin sections were cut with an ultramicrotome and stained with toluidine blue and visualized by optical microscopy. Then, the tissue was cut into ultra-thin sections with an ultra-thin slicer and stained with $2 \%$ uranyl acetate and lead citrate. After drying, the ultrastructure of the testes was observed and photographed under TEM.

\section{Statistical analysis}

Statistical analyses were performed using SAS v8.02 software (SAS Institute Inc., Cary, NC, USA). Continuous data are presented as the mean $\pm \mathrm{SD}$, and categorical data are presented as a percentage. One-way analysis of variance (ANOVA) was performed for the parametric data. Comparisons of numerical data between any 2 groups were performed using the student's $t$ test. Categorical data were compared using the chi-square test. A $p<0.05$ was considered statistically significant.

\section{Results}

\section{Effect of CS on rat body weight}

The body weights before and after the treatment are shown in Table 1. There were no significant differences among the 6 groups in pre-treatment weight. Compared with the control group, the final body weight of the CP group was significantly reduced.

\section{Effect of CS on TW, ESC, SM and ST in SD rats}

The testicle weights in all 6 groups are shown in Table 2. Compared with the control group, the testicle weight, epididymal sperm count, and sperm motility in the $\mathrm{CP}$ group were significantly different $(p<0.05)$. Compared with the CP group, there were significant differences in these factors observed in the $\mathrm{CP}+\mathrm{LCS}, \mathrm{CP}+\mathrm{MCS}$, $\mathrm{CP}+\mathrm{HCS}$ and $\mathrm{CP}+\mathrm{VitE}$ groups $(p<0.05$, Figure $1)$. There were no significant differences in serum testosterone among the 6 groups $(p>0.05$, Figure 1). Microscopic changes were observed under the microscope (200X, HE staining, Figure 2).

Effect of CS on the expression of Thy1, Oct4, $P L Z F, C-k i t$ and GDNF in rat testis tissue The qPCR results revealed a significant difference in Thy1, Oct4, PLZF and GDNF expression between the $\mathrm{CP}$ group and $\mathrm{CP}+\mathrm{LCS}$ group (Figure 3 and Table 3, $p<0.05$ ), but there was no significant difference in $\mathrm{C}$-kit between the two

Table 1. Primers for Thy1, Oct4, PLZF, C-kit and GDNF genes

\begin{tabular}{lllc}
\hline Gene & Forward primer (5'-3') & Reverse primer (3'-5') & Length (bp) \\
\hline$\beta$-actin & AGAGGGAAATCGTGCGTGAC & GCCGGACTCATCGTACTCCT & 463 \\
\hline Thy-1 & GCAGAGGTCTTGCTTCTCCCG & CACAGTCCAACTTCCCTCATCCATATCT & 369 \\
\hline PLZF & TGGTGCCTCCAGCCACATAACCA & GCTCAGCTTGCTTTACAGTCCAGGTT & 395 \\
\hline Oct4 & TGGAGAAGGAGAAGCTGGAGCAAAA & GGCAGATGGTCGTTTGGCTGAATA & 186 \\
\hline c-kit & TTACTCCAACTTGGCAAACTGCAACC & TTTCACCCCGCCCTTCCCTTCT & 450 \\
\hline GDNF & TCCTGACCAGTTTGATGACG & CTCTGCGACCTTTCC & 158 \\
\hline
\end{tabular}


Table 2. Effects of CP, CS and VitE on Initial body weight (IBW, g), Final body weight (FBW, g), Testicle weight (TW, g), Epididymal sperm count (ESC, 106/ml), Sperm motility (SM, \%), and Serum testosterone (ST, ng/ml)

\begin{tabular}{lcccccc}
\hline Group & Control & CP & CP+LCS & CP+MCS & CP+HCS & CP+VitE \\
\hline IBW & $216.7 \pm 4.1$ & $216.2 \pm 2.4$ & $213.7 \pm 3.1$ & $217.3 \pm 6.8$ & $214.1 \pm 6.7$ & $213.6 \pm 3.9$ \\
\hline FBW & $242.9 \pm 9.6$ & $174.5 \pm 11.0^{*}$ & $209.3 \pm 11.2^{\#}$ & $217.2 \pm 11.6^{\#}$ & $214.4 \pm 10.6^{\#}$ & $201.0 \pm 8.4^{\#}$ \\
\hline TW & $1.9 \pm 0.1$ & $1.2 \pm 0.1^{*}$ & $1.8 \pm 0.1^{\#}$ & $1.8 \pm 0.1^{\#}$ & $1.8 \pm 0.1^{\#}$ & $1.8 \pm 0.1^{\#}$ \\
\hline ESC & $86.3 \pm 4.3$ & $7.9 \pm 3.4^{*}$ & $79.3 \pm 4.0^{\#}$ & $81.5 \pm 3.0^{\#}$ & $79.3 \pm 3.8^{\#}$ & $74.4 \pm 3.0^{\#}$ \\
\hline SM & $49.1 \pm 3.1$ & $4.8 \pm 3.7^{*}$ & $43.4 \pm 3.4^{\#}$ & $48.3 \pm 2.8^{\#}$ & $45.9 \pm 3.8^{\#}$ & $44.1 \pm 4.3^{\#}$ \\
\hline ST & $61.4 \pm 5.3$ & $59.8 \pm 4.5$ & $64.0 \pm 6.1$ & $62.2 \pm 2.5$ & $58.1 \pm 5.3$ & $62.0 \pm 8.0$ \\
\hline
\end{tabular}

All the data were expressed as the mean $\pm \mathrm{SD}(\mathrm{n}=6)$. * Significant difference compared with the control group $(\mathrm{p}<0.05)$; \# significant difference compared with the CP group $(\mathrm{p}<0.05)$.

groups $(p>0.05)$. The western blot results were consistent with the qPCR data (Figure 4).

\section{CS has a therapeutic effect on the damage caused by CP as observed under TEM}

The changes in testicular spermatogonia were observed under TEM. The chromatin of spermatogonial nucleoli in the CP group was fragmented, coagulated and atrophied, mitochondria were enlarged, internal sputa were absent, and a large number of autophagic vacuoles were observed in the cytoplasm (Figure 5A). The spermatogonial cells in the CP+LCS group showed a uniform nucleus, clear nucleoli, and abundant mitochondria (Figure 5B). Therefore, CS has a therapeutic effect on the damage to spermatogonial spermatogenic ability caused by $\mathrm{CP}$.

\section{Discussion}

The incidence of infertility has increased annually, and the current worldwide incidence of infertility has reached $15 \%$ (7). The proportion of male factors contributing to this problem is approximately 50\% (8). Semen analysis indicators are now used to assess male fertility, and the main reasons for infertility are reduced sperm number and decreased sperm viability. Although the etiology of infertility involves genetic, endocrine, immune and environmental factors, there is still a lack of effective drug treatment to improve sperm count and viability (9).

Traditional Chinese medicine has unique properties for treating oligospermia and asthenospermia. The recent application of modern biological technology has allowed for the evaluation of many herbs in the reproductive system. CS is a traditional kidney medicine with significant positive effects and adequate kidney Qi that can lead to improved semen quality. Other studies have demonstrated that CS increases cell proliferation and neuroblast differentiation in the dentate gyrus of mice by reducing serum corticosterone levels and increasing BDNF levels in this area (10). Wang $X$ et al. demonstrated that compounds from CS exhibit phytoestrogenic and phytoandrogenic activities (11). CS exhibits in vitro and in vivo pharmacological activities and reduces fatigue, hypoxia, oxidation, diabetic symptoms, and viral activity and can modulate the immune system (12). The data from the study by Liu et al. demonstrated important anti-aging effects of CS (13).

Studies of CS both in and outside of China suggest that it plays an important role in the reproductive system. Abdel-Magied EM (14) reported that CS significantly increases the testicular weight of immature male Wistar rats. In immature rats, oral CS has the following effects: increased testicular sperm content and improved 

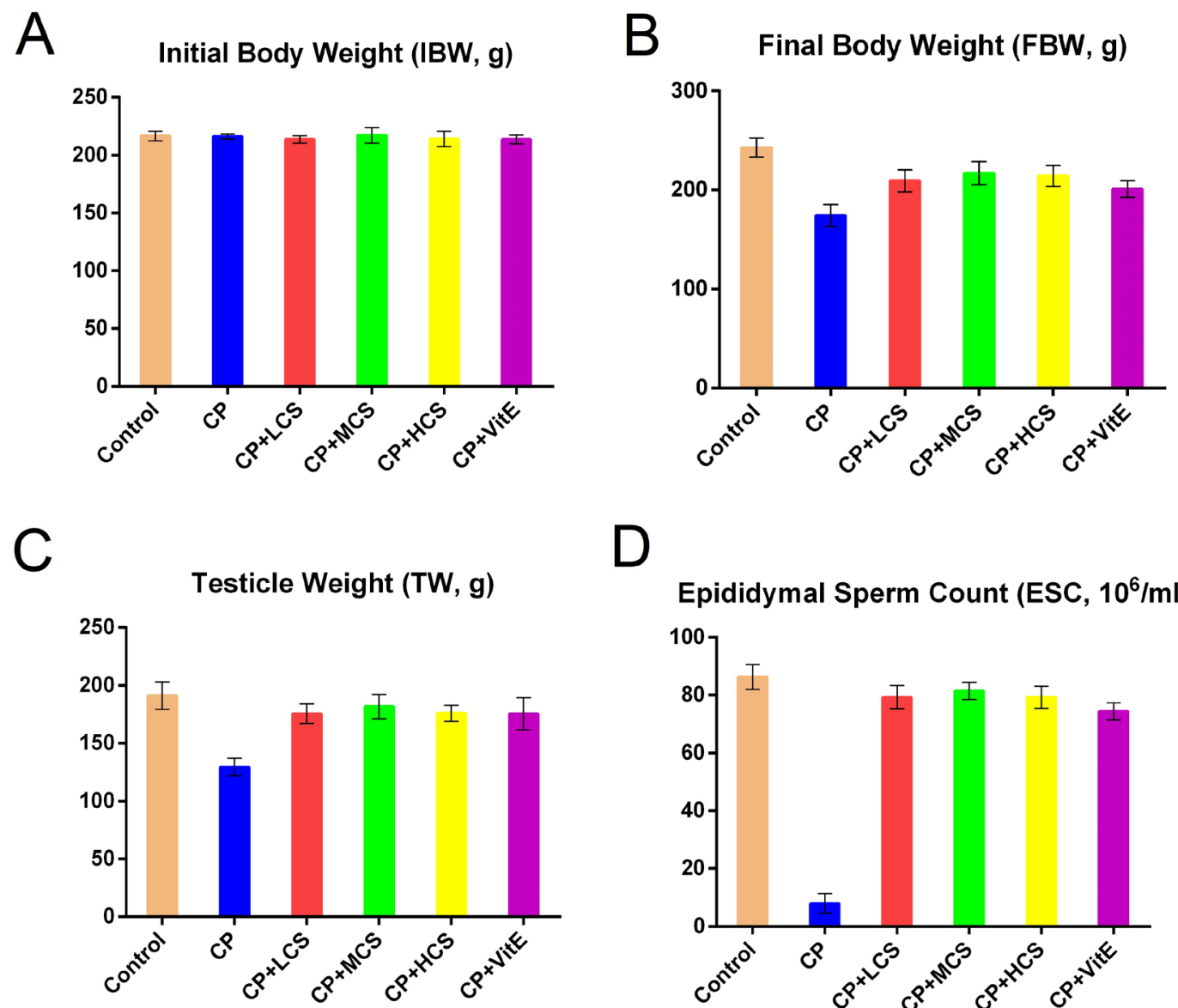

$\mathrm{D}$
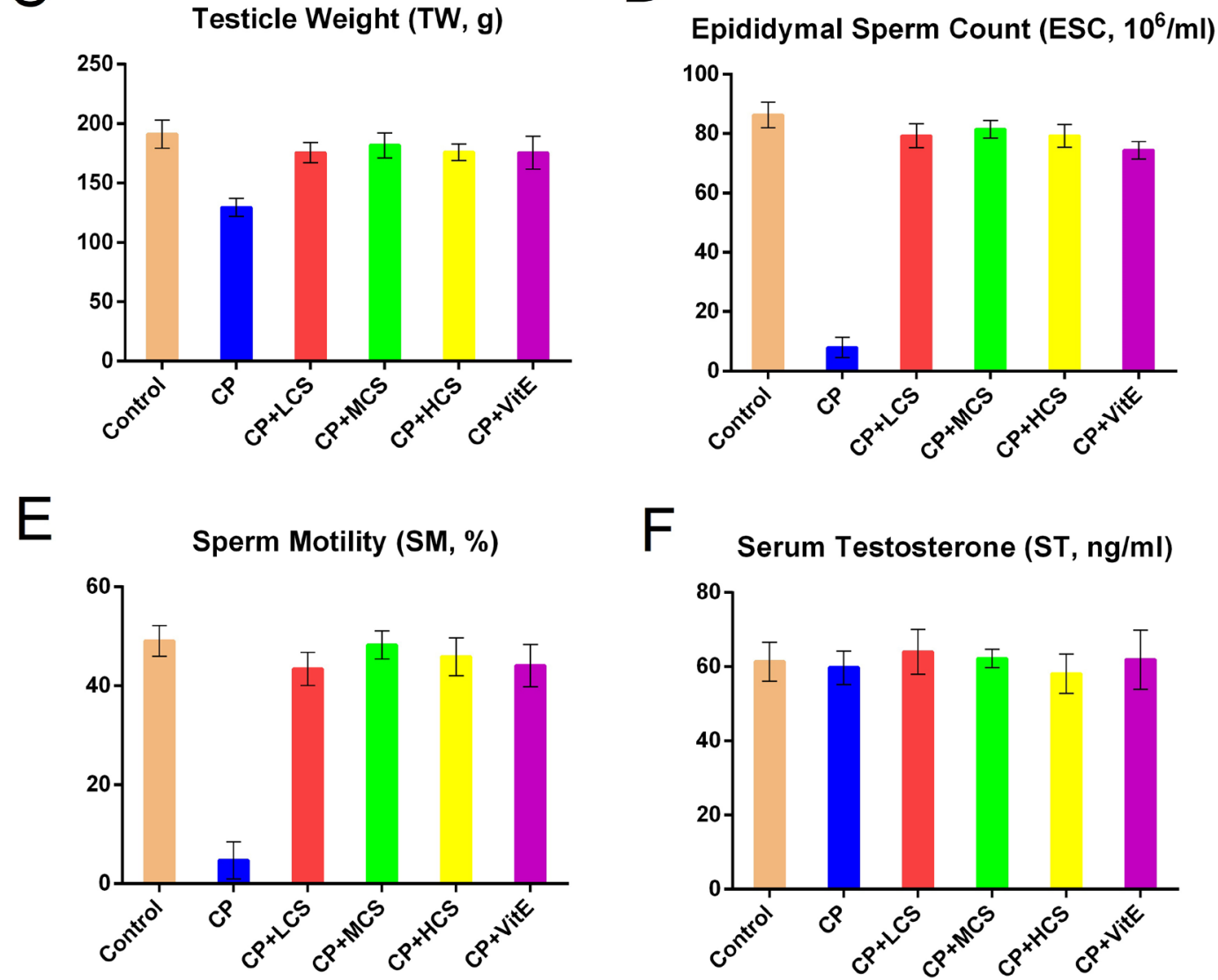

Fig. 1. Effect of CS on body weight, testicle weight, epididymal sperm count, sperm motility and serum testosterone in SD rats

Effects of CP, CS and VitE on Initial body weight (IBW, g), Final body weight (FBW, g), Testicle weight (TW, centigram), Epididymal sperm count (SC, 106/ml), Sperm motility (SM, \%), and Serum testosterone (ST, ng/ml). There were significant differences in FBW, TW, ESC and $\mathrm{SM}$ between the $\mathrm{CP}$ group and control group and between the $\mathrm{CP}$ group and the $\mathrm{CP}+\mathrm{LCS}, \mathrm{CP}+\mathrm{MCS}, \mathrm{CP}+\mathrm{HCS}, \mathrm{CP}+\mathrm{VitE}$ groups $\left(\mathrm{p}<0.05\right.$, ${ }^{*}$. 

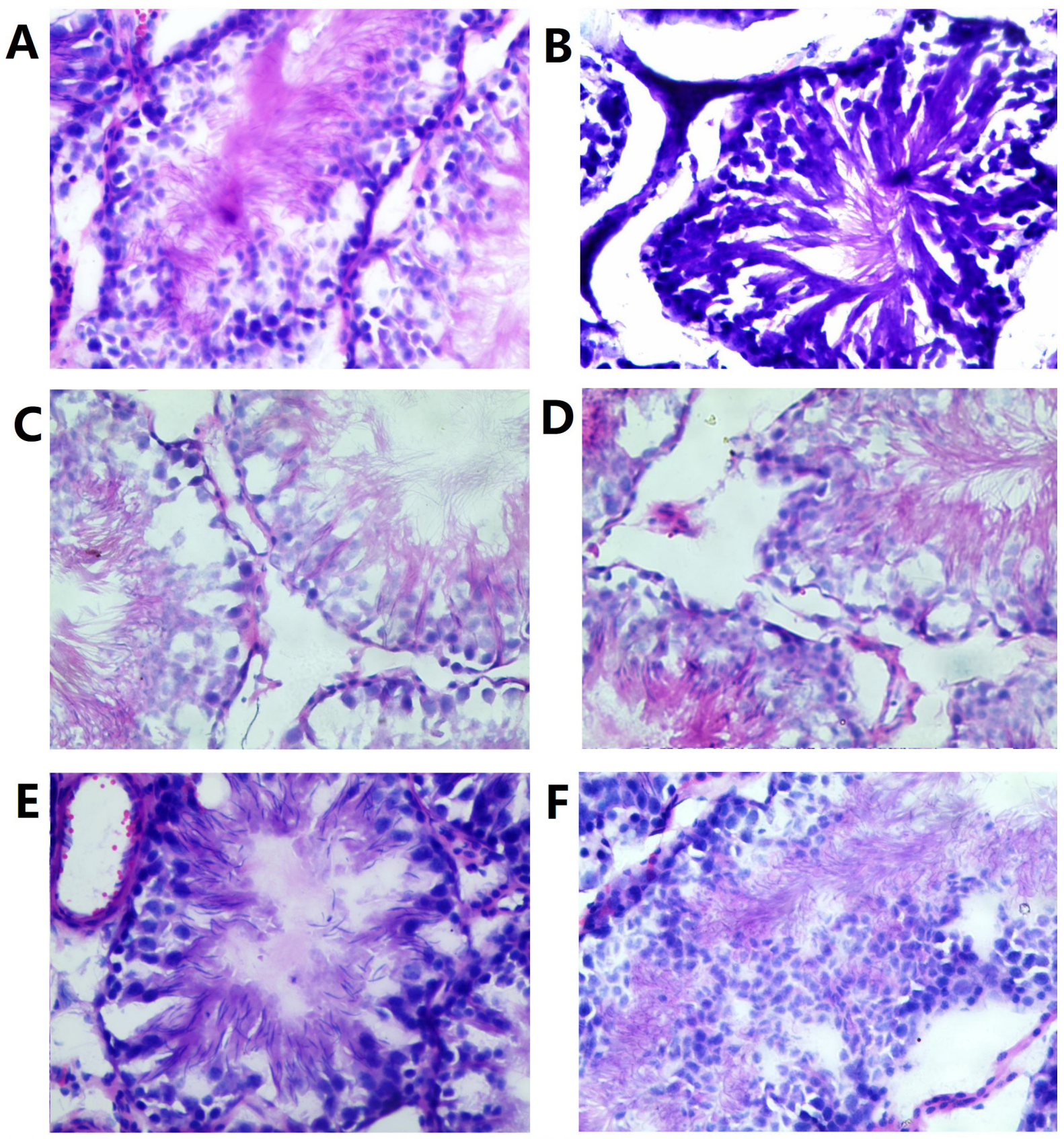

Fig. 2. Microscopic changes of rat testicle

Microscopic changes of rat testicle. A-F: control group, CP group, CP+LCS, CP+MCS, CP+HCS and CP+VitE groups (HE staining, 400X). In the control group, normal seminiferous tubules and spermatogenic cells were observed at all levels. In the CP group, it can be observed that spermatogenic cells of various levels shrink in different degrees and the structure is unclear. The seminiferous tubules and spermatogenic cells at all levels were clearly discernible in the CS or VitE group. 
Table 3. qPCR results between the $\mathrm{CP}$ and $\mathrm{CP}+\mathrm{LCS}$ groups

\begin{tabular}{lcccc}
\hline $\boldsymbol{\Delta C t}$ & Group CP & Group CP+LCS & t ratio & $\boldsymbol{p}$ value \\
\hline Thy1 & $5.37 \pm 1.66$ & $15.68 \pm 3.49$ & 4.62 & $0.0099^{*}$ \\
\hline Oct4 & $6.65 \pm 1.06$ & $15.16 \pm 2.46$ & 5.50 & $0.0053^{*}$ \\
\hline PLZF & $4.78 \pm 1.05$ & $14.10 \pm 2.77$ & 5.44 & $0.0055^{*}$ \\
\hline C-kit & $8.74 \pm 1.84$ & $10.31 \pm 2.97$ & 0.78 & 0.4789 \\
\hline GDNF & $9.76 \pm 1.46$ & $16.69 \pm 0.68$ & 7.43 & $0.0018^{*}$ \\
\hline
\end{tabular}

All data were expressed as mean $\pm S D(n=3)$. * Significant difference compared with the control group $(p<0.05) ;$ \# significant difference compared with the CP group $(p<0.05)$.

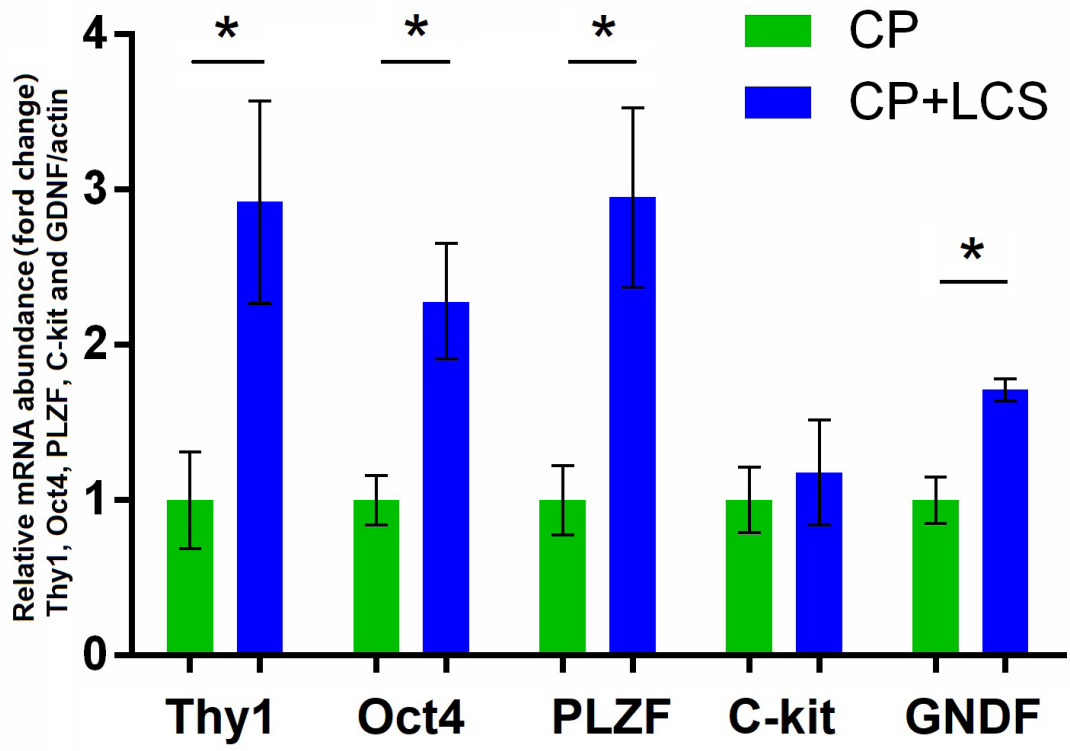

Fig. 3. qPCR results of Thy1, Oct4, PLZF, C-Kit and GDNF

The qPCR results showed significant differences in Thy 1 , Oct 4 , PLZF and GDNF $(\mathrm{p}<0.05, *)$, but there was no difference in C-Kit expression between the CP group and CP+LCS group ( $p>0.05)$.

spermatogenic tubules with sperm, increased epididymal tail sperm count, significantly increased sperm survival rate, increased sperm motility, and reduced abnormal sperm count. The Korean scholar Yang WM also reported similar findings in which oral CS increased glial cell line-derived neurotrophic factor mRNA and protein expression levels (3). The upregulation of GDNF can increase both the number of rat epididymal sperm and absolute testicular weight, reduce the number of abnormal spermatozoa, and increase the percentage of active sperm (4). Chinese scholars have reported that CS can sig- nificantly improve testosterone levels in male mice. Although several studies have suggested that CS is helpful in the reproductive system, the mechanism of action has not been elucidated. In this study, we used cyclophosphamide to construct a rat model of oligospermia and asthenospermia (control group). Our data showed that 4 weeks of treatment with different concentrations of CS did not significantly alter the weight of the CS group compared with that of the control group and blank group $(p<0.05)$. These results are inconsistent with previous studies. The differences may be due to separation of rat testicu- 


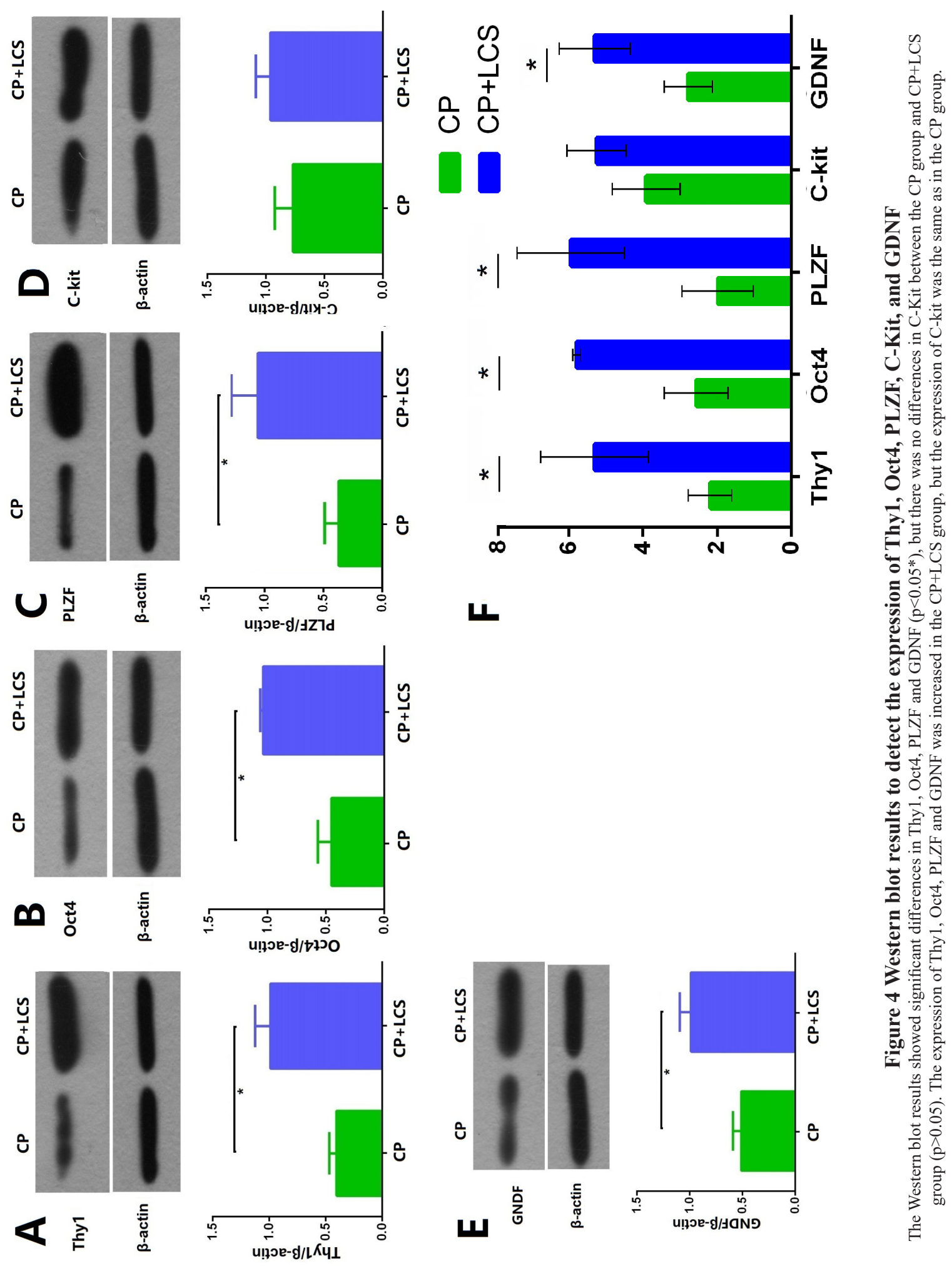




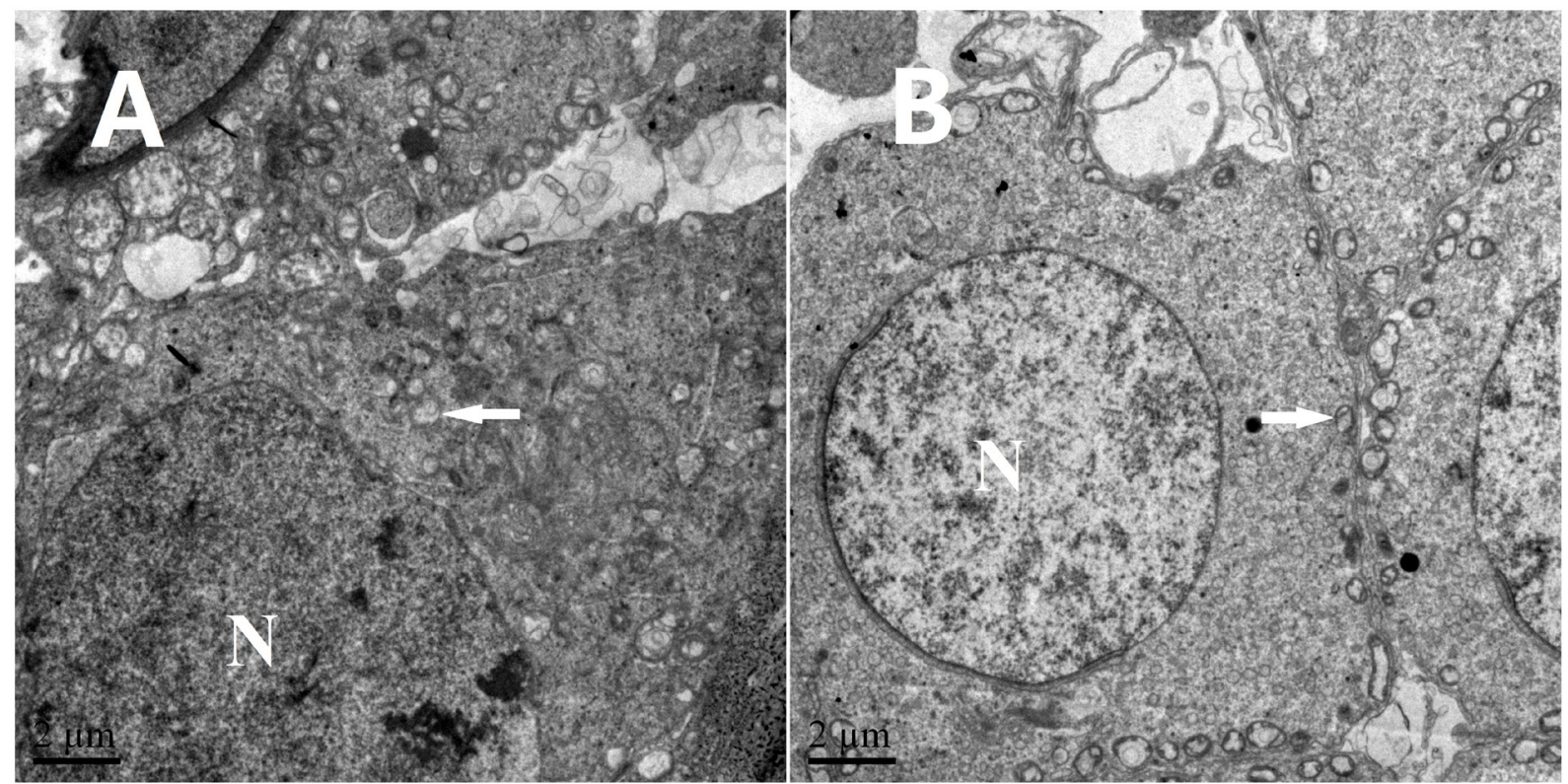

Fig. 5. TEM observations of testicular spermatogonia apoptosis

The changes in testicular spermatogonia were observed under TEM. The chromatin of the spermatogonial nucleus in the CP group was fragmented, coagulated and atrophied $(\mathrm{N})$, mitochondria were enlarged, internal sputum was absent $(\leftarrow)$, and a large number of autophagic vacuoles were observed in the cytoplasm. The spermatogonial cells in the CP+LCS group showed a uniform nucleus $(\mathrm{N})$, clear nucleoli, and abundant mitochondria $(\rightarrow)$.

lar tissue in the process. It is also difficult to obtain tissue weight. The number of spermatozoa in the epididymal tail of the CS group increased compared with that in the control group, and the difference was statistically significant $(p<0.05)$. The results of this study are consistent with the results of Yang WM suggested that CS does contribute to sperm content in the testis.

Yang WM also reported that CS may improve spermatogenesis by improving spermatogonia. The three groups of CS experimental animals showed improved spermatogenesis, and there was no obvious concentration effect between the three groups. Therefore, we used real-time PCR to detect the low concentration markers of testicular tissue spermatogonia (Oct4, Thy1, PLZF, and C-kit) (15). The results showed that the mRNA expression levels of Oct4, Thy1, and PLZF in the CS group were higher than those in the control group, and the differences were statistically significant. There was no signifi- cant change in the expression of C-kit RNA. Studies have shown that Thy1, PLZF, and Oct4 are mainly expressed in undifferentiated spermatogonial stem cells (As, Apr, Aal) (16). Conversely, the C-kit gene is mainly expressed in differentiated A1 to A4 spermatogonia and is not expressed in undifferentiated spermatogonia. These results suggest that CS mainly promoted the proliferation of undifferentiated spermatogonial stem cells and had no significant effect on spermatogonia. During the process of spermatogenesis, spermatogonia receive signals and nutritional support from Sertoli cells, which allow them to proliferate and differentiate. GDNF is indispensable for spermatogonial stem cell self-renewal through its binding to the GFRA1/ RET receptor and the subsequent effect on sperm quality (17).

The GDNF gene is expressed in all stages of mouse spermatogenesis and is mainly expressed in Sertoli cells. In this study, we found that the 
expression of GDNF mRNA in the CS low concentration group was significantly higher than that in the control group. These findings suggest that CS may promote the expression of undifferentiated spermatogonia by promoting GDNF expression (18). There are many studies on the molecular pathway of GDNF in mice and other model animals. GDNF protein specifically binds to GFR $\alpha 1$, which is expressed on undifferentiated spermatogonial cell membranes and activates tyrosine phosphorylation of Ret protein. The signal activation causes downstream MAPK-3 activation. This signaling pathway also exists in rats and suggests that CS may play a role that involves the GDNF pathway.

The sperm viability of the three CS groups was significantly higher than that of the control group $(p<0.05)$. Increases in serum testosterone improve the quality and activity of spermatozoa (19). Fatihah et al. (20) found that a CS decoction improved sperm quality by increasing serum testosterone concentration; our results are consistent with these findings. The serum concentration of testosterone in SD rats treated with CS was significantly higher than that in the control group $(\mathrm{p}<0.05)$, which suggests that CS may improve serum testosterone level and improve sperm viability.

The complexity of the CS water extract composition may lead to pharmacological diversity. Previous studies have shown that CS is rich in trace elements and has high zinc content (19). Lee et al. suggested that CS extract promotes male fertility by improving spermatogenesis in golden hamsters (21). CS decoction improves rat sperm quality. Recent studies have shown that oxidative stress is an important factor in the manufacture of weak sperm. CS decoction contains a variety of ingredients with potent antioxidant effects, and current research on the anti-oxidant elements of CS has focused on carbohydrates, flavonoids and polyphenols. These compounds are capable of scavenging oxygen free radicals. A recent study reported that two new water-soluble polysaccharides (22) and songarin A (23) were isolated from CS. Future studies should focus on the research and development of monomer components and expand their use for spermatogonia cell proliferation in vitro.

In conclusion, CS can promote spermatozoa processes in rats with oligoasthenospermia by increasing sperm count in the epididymis and improving sperm motility and has a therapeutic effect on the spermatogenic damage caused by $\mathrm{CP}$ in SD rats. However, due to the limitations of Chinese herbal medicine purification methods, the role of individual components of the CS decoction is difficult to obtain, limiting further therapeutic development of CS and other herbs.
Abbreviations
CS Cynomorium songaricum
CP Cyclophosphamide
IBW Initial body weight
FBW Final body weight
TW Testicle weight
ESC Epididymal sperm count
SM Sperm motility
ST Serum testosterone
TEM Transmission electron microscopy
GDNF Glial cell line-derived neurotrophic fac- tor
SPF Skatepark Formula ${ }^{\mathrm{TM}}$
LCS low $(0.5 \mathrm{~g} / \mathrm{kg} \cdot$ day $)$ concentrations of CS
MCS Medium $(1 \mathrm{~g} / \mathrm{kg} \cdot$ day $)$ concentrations of CS
HCS High $(2 \mathrm{~g} / \mathrm{kg} \cdot$ day $)$ concentrations of CS
HE staining Hematoxylin-eosin staining
EP tube Eppendorf tube
GAPDH glyceraldehyde-3-phosphate
dehydrogenase
PVDF polyvinylidene fluoride
SD Sprague Dawley® Rats 


\section{Authors' contribution}

Xiaoxiao Han (Data curation)

Rongsheng Zhou (Methodology; Software; Supervision)

Wei Zheng (Investigation)

Xitao Wang (Data curation)

Shuyan Mao (Pathology)

Zhenbei Li (Software)

Zhiguo Zhang (Data curation)

Lin Hao (Software)

Zhenduo Shi (Data curation)

Bo Chen (Data curation)

Yijuan Cao (Data curation)

Dianjun Yu (Formal analysis)

Kun Pang (Writing - original draft; Writing - review \& editing)

Conghui Han (Funding acquisition)

\section{Acknowledgements}

1. We would like to thank American Journal Experts (AJE) for providing the high-quality English editing service for this article.

2. This study was funded by funds as follows: National Natural Science Fund (81774089); Jiangsu Province, the medical innovation team (CXTD-2016-48); Jiangsu Province, key research and development program (BE2017635); Jiangsu Province, young medical talents (QNRC2016386); Jiangsu Province, natural science research projects (17KJB360001); Jiangsu Provincial Traditional Chinese Medicine Bureau of Science and Technology Project (YB2017055); Ningbo Natural Science Foundation (2017A610194).

Conflict of interest statement

The authors declare no conflict of interest in preparing this article

Ethics and consent statements

The study was provided by the Experimental Animal Center of Xuzhou Medical University (SYXK (Su) 2017-0011).

\section{Reference}

1. Chyra-Jach D, Kaletka Z, Dobrakowski M, Machoń-Grecka A, Kasperczyk S, Birkner E, et al. The Associations between Infertility and Antioxidants, Proinflammatory Cytokines, and Chemokines. Oxid Med Cell Longev. 2018:8354747. DOI: 10.1155/2018/8354747

2. Yang Y, Yi X, Peng M, Zhou Y. Stable carbon and nitrogen isotope signatures of root-holoparasitic Cynomorium songaricum and its hosts at the Tibetan plateau and the surrounding Gobi desert in China. Isotopes Environ Health Stud. 2012;48(4):483-93. DOI: 10.1080/10256016.2012.680593

3. Yang WM, Kim HY, Park SY, Kim HM, Chang MS, Park SK. Cynomorium songaricum induces spermatogenesis with glial cell-derived neurotrophic factor (GDNF) enhancement in rat testes. J Ethnopharmacol. $2010 \mathrm{Apr}$ 21;128(3):693-6. DOI: 10.1016/j.jep.2010.02.020

4. Abd el-Rahman HA, el-Badry AA, Mahmoud OM, Harraz FA. The effect of the aqueous extract of Cynomorium coccineum on the epididymal sperm pattern of the rat. Phytother Res. 1999 May;13(3):248-50. DOI: 10.1002/(SICI)1099-1573(199905)13:3<248::AIDPTR425>3.0.CO;2-D

5. Cao YJ, Li ZB, Qi YJ, Liu Y, Gu J, Hu FF, et al. [Cynomorium songaricum improves sperm count and motility and serum testosterone level and promotes proliferation of undifferentiated spermatogonia in oligoasthenospermia rats]. Zhonghua Nan Ke Xue. 2016 Dec;22(12):1116-21.

6. Ceribasi AO, Turk G, Sonmez M, Sakin F, Atessahin A. Toxic effect of cyclophosphamide on sperm morphology, testicular histology and blood oxidant-antioxidant balance, and protective roles of lycopene and ellagic acid. Basic Clin Pharmacol Toxicol. 2010 Sep;107(3):730-6. DOI: 10.1111/j.1742-7843.2010.00571.x

7. Thoma ME, McLain AC, Louis JF, King RB, Trumble AC, Sundaram R, et al. Prevalence of infertility in the United States as estimated by the current duration approach and a traditional constructed approach. Fertil Steril. 2013 Apr;99(5):1324-31.e1. DOI: 10.1016/j. fertnstert.2012.11.037

8. Skinner TAA, Ho L, Touma NJ. Study habits of Canadian urology residents: Implications for development of a competence by design curriculum. Can Urol Assoc J. 2017 Mar-Apr;11(3-4):83-7. DOI: 10.5489/cuaj.4132

9. Silberstein T, Har-Vardi I, Harlev A, Friger M, Hamou B, 
Barac T, et al. Antioxidants and Polyphenols: Concentrations and Relation to Male Infertility and Treatment Success. Oxid Med Cell Longev. 2016;2016:9140925. DOI: 10.1155/2016/9140925

10. Yoo DY, Choi JH, Kim W, Jung HY, Nam SM, Kim JW, et al. Cynomorium songaricum extract enhances novel object recognition, cell proliferation and neuroblast differentiation in the mice via improving hippocampal environment. BMC Complement Altern Med. 2014 Jan 7;14:5. DOI: 10.1186/1472-6882-14-5

11. Wang X, Tao R. Compounds from Cynomorium songaricum with Estrogenic and Androgenic Activities Suppress the Oestrogen/Androgen-Induced BPH Process. Evid Based Complement Alternat Med. 2017; 6438013. DOI: 10.1155/2017/6438013

12. Cui Z, Guo Z, Miao J, Wang Z, Li Q, Chai X, et al. The genus Cynomorium in China: an ethnopharmacological and phytochemical review. J Ethnopharmacol. 2013;147(1):1-15. DOI: 10.1016/j.jep.2013.01.020

13. Liu HP, Chang RF, Wu YS, Lin WY, Tsai FJ. The Yang-Tonifying Herbal Medicine Cynomorium songaricum Extends Lifespan and Delays Aging in Drosophila. Evid Based Complement Alternat Med: eCAM. 2012;2012:735481. DOI: 10.1155/2012/735481

14. Abdel-Magied EM, Abdel-Rahman HA, Harraz FM. The effect of aqueous extracts of Cynomorium coccineum and Withania somnifera on testicular development in immature Wistar rats. J Ethnopharmacol. 2001;75(1):1-4. DOI: 10.1016/S0378-8741(00)003482

15. Pesce M, Wang X, Wolgemuth DJ, Scholer H. Differential expression of the Oct-4 transcription factor during mouse germ cell differentiation. Mech Dev. 1998;71(12):89-98. DOI: 10.1016/S0925-4773(98)00002-1

16. Costoya JA, Hobbs RM, Barna M, Cattoretti G, Manova K, Sukhwani M, et al. Essential role of Plzf in maintenance of spermatogonial stem cells. Nat Genet. 2004;36(6):653-9. DOI: 10.1038/ng1367

17. Takashima S, Kanatsu-Shinohara M, Tanaka T, Morimoto $\mathrm{H}$, Inoue $\mathrm{K}$, Ogonuki $\mathrm{N}$, et al. Functional differences between GDNF-dependent and FGF2-dependent mouse spermatogonial stem cell self-renewal. Stem cell reports. 2015;4(3):489-502. DOI: 10.1016/j.stemcr.2015.01.010

18. Morimoto H, Kanatsu-Shinohara M, Takashima S, Chuma S, Nakatsuji N, Takehashi M, et al. Phenotypic plasticity of mouse spermatogonial stem cells. PloS one. 2009;4(11):e7909. DOI: 10.1371/journal. pone.0007909

19. Oteiza PI, Olin KL, Fraga CG, Keen CL. Zinc deficiency causes oxidative damage to proteins, lipids and DNA in rat testes. J Nutr. 1995;125(4):823-9.

20. Fatihah SN, Safiah J, Abol-Munafi AB, Ikhwanuddin M. Effect of testosterone undecanoate hormone on sperm and its level in the hemolymph of male mud spiny lobster, Panulirus polyphagus. Pak J Biol Sci. 2014;17(7):937-41. DOI: 10.3923/pjbs.2014.937.941

21. Lee JS, Oh HA, Kwon JY, Jeong MH, Lee JS, Kang DW, et al. The Effects of Cynomorium songaricum on the Reproductive Activity in Male Golden Hamsters. Dev Reprod2013;17(1):37-43. DOI: 10.12717/ DR.2013.17.1.037

22. Tuvaanjav S, Shuqin H, Komata M, Ma C, Kanamoto $\mathrm{T}$, Nakashima $\mathrm{H}$, et al. Isolation and antiviral activity of water-soluble Cynomorium songaricum Rupr. polysaccharides. J Asian Nat Prod Res. 2016;18(2):159-71. DOI: $10.1080 / 10286020.2015 .1082547$

23. Xie SA, Li GY, Huang J, Zheng YX, Wang HY, Zhang $\mathrm{K}$, et al. A new flavanol from Cynomorium songaricum. J Asian Nat Prod Res. 2013;15(4):413-6. DOI: 10.1080/10286020.2013.774765 